\title{
The statistics of BAT-to-XRT flux ratio in GRB: Evidence for a characteristic value and its implications
}

\author{
D. Kazanas ${ }^{1}$, J. Racusin ${ }^{1}$, J. Sultana ${ }^{2}$, and A. Mastichiadis ${ }^{3}$
}

Demos.Kazanas@nasa.gov

Received —

\footnotetext{
${ }^{1}$ Astrophysics Science Division, NASA/Goddard Space Flight Center, Greenbelt, MD 20771 USA.

${ }^{2}$ Mathematics Department, Faculty of Science, University of Malta, Msida MSD2080 Malta.

${ }^{3}$ Department of Physics, University of Athens, Panepistimiopolis, GR 15783, Zografos, Greece
} 


\begin{abstract}
We present the statistics of the ratio, $\mathcal{R}$, between the prompt and afterglow "plateau" fluxes of GRB. This we define as the ratio between the mean prompt energy flux in Swift BAT and the Swift XRT one, immediately following the steep transition between these two states and the beginning of the afterglow stage referred to as the "plateau". Like the distribution of many other GRB observables, the histogram of $\mathcal{R}$ is log-normal with maximum at a value $\mathcal{R}_{m} \simeq 2,000$, FWHM of about 2 decades and with the entire distribution spanning about 5 decades in the value of $\mathcal{R}$. We note that the peak of the distribution is close to the proton-to-electron mass ratio $\left(\mathcal{R}_{m} \simeq m_{p} / m_{e}=1836\right)$, as proposed to be the case in an earlier publication, on the basis of a specific model of the GRB dissipation process. It therefore appears that, in addition to the values of the energy of peak luminosity $E_{\mathrm{pk}} \sim m_{e} c^{2}$, GRB present us with one more quantity with an apparent characteristic value. The fact that the values of both these quantities $\left(E_{\mathrm{pk}}\right.$ and $\left.\mathcal{R}\right)$ are consistent with the same specific model invoked to account for the efficient conversion of their relativistic proton energies to electrons, argues favorably for its underlying assumptions.
\end{abstract}

Subject headings: cosmological parameters — Gamma-ray burst: general 


\section{Introduction}

Gamma-Ray Bursts (GRB), $\gamma$-ray emission events at cosmological distances by relativistically moving (of Lorentz factors $\Gamma \sim 200$ ) plasmas, remain enigmatic despite much observational and theoretical progress over the past twenty five years. Furthermore they present us with new puzzles each time instrumentation and ensuing observations that measure any of their attributes improve significantly. The most recent such puzzle is that of the shape of their afterglow light curves as determined by Swift, the subject of the present note.

While the association of GRB with RBW is generally accepted, there are still serious gaps in our fundamental understanding of their underlying physics. For instance, the presence of the GRB relativistic outflows with the necessary Lorentz factors requires flows with energy-to-mass ratios larger than $\sim 100$, a condition not naturally encountered in most astrophysical plasmas. Similarly, bearing in mind that in a RBW the proton to electron postshock pressure ratios are roughly in proportion to that of their mass, i.e. $m_{p} / m_{e} \sim 2000$, one would expect a very small radiative efficiency $(\eta \sim 1 / 2000)$ for these shocks, considering that protons are generally inefficient radiators. This issue is usually resolved by assuming the efficient transfer of energy from protons to electrons to keep the two components in rough pressure equipartition, thus guaranteeing the efficient emission of radiation by the synchrotron emitting electrons. Another open issue associated with the GRB prompt stage is the rather limited range of the energy of peak luminosity $E_{\mathrm{pk}} \sim 0.3$ $\mathrm{MeV}$ (extending on occasions to a few $\mathrm{MeV}$ ), intriguingly close to the electron rest mass on the Earth frame, but not on the GRB rest frame, considering the large Lorentz factors of their blast waves. This characteristic energy is the defining attribute of the GRB prompt emission since emission at this energy declines very fast GRB develop in time and enters their, less variable, afterglow stage.

Since their discovery by BeppoSAX, GRB afterglows have been considered a distinct and separate phase of the GRB phenomenon. However, to the best of our knowledge, there is no formal criterion for the transition from the prompt to the afterglow GRB phase; it is generally considered that the prompt GRB emission continues until the RBW has reached its deceleration radius $R_{D}$, the radius at which the RBW has swept-up mass-energy $M c^{2} \simeq m_{p} c^{2} n R_{D}^{3} \simeq E / \Gamma^{2}$ ( $E$ is total energy of the RBW, $n$ the circumburst density assumed to be constant - and $\Gamma$ its asymptotic Lorentz factor). Beyond this radius, the GRB Lorentz factor $\Gamma$ decreases gradually (e.g. $\Gamma \propto R^{-3 / 2}$ for adiabatic evolution in a uniform density medium) as the added inertia of the swept-up medium exceeds the value that would permit expansion at constant $\Gamma$. The afterglow emission, just as that of the prompt phase, is considered to be synchrotron radiation by shock accelerated electrons, in rough equipartition with the protons of the postshock region. Under these assumptions, one can compute for the GRB afterglow stage the resulting spectrum and its time evolution. The computation of the GRB X-ray flux evolution in time was first performed by Sari et al. (1998) for spherically symmetric outflows, and by Sari et al. (1999) for jet-like outflows, assuming the spectrum of electrons injected at the shock to be $d N / d E \propto E^{-p}, p \gtrsim 2$. The 
resulting X-ray light curves were, then, shown to be power laws in time, $F_{X} \propto t^{-\alpha}, \alpha \gtrsim 1$, in broad agreement with the sparsely sampled afterglow light curves of the pre-Swift era.

The launch of Swift and its ability to follow closely the evolution of GRBs from their prompt ( $\gamma$-ray emitting) to the afterglow (X-ray emitting) stages provided yet another set of unexpected, puzzling facts, grossly inconsistent with expectations based on the models described above (Tagliaferri et al. 2005; Nousek et al. 2006; Zhang et al. 2006; Evans et al. 2009): Instead of the predicted decrease of their X-ray flux as $L_{X} \propto t^{-\alpha}, \alpha \gtrsim 1$, the decrease is much steeper $(\alpha \sim 3-6)$, followed either $(i)$ by a much shallower section (referred to as the "plateau") $(\alpha \sim 0)$, which is succeeded for $t>T_{\text {brk }} \sim 10^{3}-10^{5}$ sec by a decline of $\alpha \simeq 1$ or $(i i)$ not by a plateau, but by a more conventional decline $(\alpha \simeq 1)$ (these light curves exhibit also occasional large amplitude flares which we will not discuss at present).

The commonly accepted account of the steep light curve decline segment is that of high latitude late emission (see Zhang 2007, §3.1.1 and references therein), even though the time evolution of such emission, while steeper $(\alpha \simeq 2)$ than that of Sari et al. (1998), it is still less steep than what is observed and certainly at odds with declines as steep as $t^{-6}$. However, Genet \& Granot (2009) suggested that this process can account for power laws steeper than 2 and applied to fit the steep decay light curves of a sample of 12 GRBs (Willingale et al. 2009) as steep as $t^{-3.5}$. An alternative explanation attributes this to the form of the underlying electron distribution function (Kazanas et al. 2007; Giannios \& Spitkovsky 2009), while Petropoulou et al. (2011), by adjusting the maximum energy $\gamma_{\max }$ of the electron distribution, interpret the steep decline segment as synchrotron emission by the fast cooling, high energy cutoff of the electron distribution function and the "plateau" segment to inverse Compton by its more slowly varying low energy section.

The plateau segment, because it follows that of the very steep flux decline, gives the impression of a distinct and completely separate emission from that of the GRB prompt phase. For this reason, it was proposed (not unreasonably) to represent an additional injection of energy by the GRB central source, separate from that producing the shorter but brighter prompt emission (Willingale \& O'Brien 2007). Along similar lines, Gompertz et al. (2014) even specify this additional injection as due to the propeller effect of an underlying magnetar that powers the entire burst. Anyway, these attempts to account for the behavior of the GRB afterglow light curves were devised to model these specific features, without any reference to or consideration of the broader properties of the entire burst. Several treatments have focused on just the properties of the plateau phase itself. Of these we mention those of Lei et al. (2011) and Shen \& Matzner (2012) who, by fitting the spectro-temporal evolution of the plateau segment of the light curves of several GRB, conclude that this emission takes place before the RBW has reached its deceleration radius $R_{D}$.

An altogether different approach to the afterglow evolution has been that of Sultana, Kazanas \& Mastichiadis (2013). This is different in that the afterglow evolution, including all its details, is produced as an integral part of the evolution of the entire burst, beginning with the accelerating phase of the RBW and continuing with its dissipation and 
prompt emission, including also the correct value the energy of the GRB prompt phase emission, $E_{\mathrm{pk}}$. The central notion of this model is a radiative instability that converts the relativistic proton energy behind the RBW forward shock to $e^{+} e^{-}$-pairs through the $p \gamma \rightarrow p e^{+} e^{-}$reaction; the pairs then produce more synchrotron photons, which produce more pairs and so on (Kazanas et al. 2002; Mastichiadis \& Kazanas 2006, 2008, 2009). This instability requires that the column of relativistic protons in the postshock region be larger than a critical value, in direct analogy with a supercritical nuclear pile (hence the nomenclature of the model; put simply, one cannot accumulate arbitrarily large columns of relativistic protons for the same reason that one cannot accumulate arbitrarily large amounts of $\mathrm{U}^{235}$ : they explode!). However, besides this condition on the proton column, the instability imposes a kinematic constraint on the synchrotron photon energy because the synchrotron photons emitted by the $e^{+} e^{-}$-pairs must be able to pair-produce in collisions with the protons. This effectively requires the RBW Lorentz factor to be larger than a critical value $\Gamma_{c}^{5} b \simeq 1$, a demand imposed by the kinematic threshold of the above reaction ( $b=B / B_{\text {cr }}$ is the postshock value of the GRB magnetic field with $B_{\text {cr }}=4.4 \times 10^{13} \mathrm{G}$ the value of the critical magnetic field). Incidentally, the energy of peak emission of the prompt phase of this model, on the Earth frame, is also (in units of $m_{e} c^{2}$ ) $E_{\mathrm{pk}} \simeq \Gamma^{5} b \simeq 1$, so this process provides also a reason for the observed value of $E_{\mathrm{pk}}$ of the prompt GRB phase.

One of the crucial elements of this model is the upstream scattering of the RBW synchrotron radiation and its re-interception by it. As noted in Mastichiadis \& Kazanas (2008, 2009) and more specifically in Sultana, Kazanas \& Mastichiadis (2013, hereafter SKM13) this process induces a radiation reaction on the RBW and causes a (relatively) small $(\sim 30 \%-50 \%)$ reduction of its Lorentz factor over a radius $\Delta R \lesssim R$. Even though small, this reduction pushes $\Gamma$ below the threshold value $\Gamma_{c}$ and hence arrests the transfer of energy from the RBW relativistic protons into $e^{+} e^{-}$-pairs. As noted in SKM13 this results in an abrupt reduction of the RBW radiative flux (the steep decline) and the GRB enters its afterglow stage. The reduction in flux should be by a factor roughly equal to the proton-to-electron mass ratio, i.e. by $\sim m_{p} / m_{e} \simeq 2000$, since the emitted radiation now comes from the cooling of only the electrons swept-up by the RBW. For the reasons explained in the paragraph above, the RBW continues its expansion at the new, constant, value of $\Gamma$ (until it reaches its deceleration radius) by emitting radiation only by the newly swept-up electrons. Because of the constancy of the Lorentz factor in this stage, depending on the rate of decrease of the RGW magnetic field with radius or the density profile of the ambient medium (Shen \& Matzner 2012), the ensuing synchrotron emission could be constant, decreasing or even increasing with time. Finally, beyond the deceleration radius, which occurs at time $T_{\text {brk }}$, a more conventional traditional decrease in its flux ensues, consistent with the standard models.

Recently, an interpretation of the afterglow idiosyncrasies, similar in some respects to that of SKM13, suggested by Duffell \& McFadven (2014), who proposed that a "top-heavy" jet would produce behavior similar to that observed. The main point behind this approach is, in essence, similar to that of our model (SKM13), namely a change in the Lorentz factor of the RBW on a radial scale $\Delta R$ of order or shorter than the local RBW radius, 
but much shorter than its deceleration radius $R_{D}$. The difference of this model from that of SKM13 is that this change in $\Gamma$ is achieved by adding inertia (rather than negative momentum as in SKM13) onto the RBW over a distance $\Delta R \lesssim R$, before it has reached its deceleration radius $R_{D}$. Just like in SKM13 the RBW achieves a new Lorentz factor $\Gamma^{\prime}(<\Gamma)$ and expands at this value until it piles-up enough inertia to continue its decrease at the conventional rate. One should note here that, in distinction with the model of SKM13, among other things, this model at its present stage does not imply a specific value for the ratio $\mathcal{R}$ of GRB luminosities between their prompt and plateau stages.

The specific value of the ratio of the GRB fluxes between their prompt phase and the afterglow plateau, implied by the model put forward by SKM13, gave us the impetus to compile and present in this paper the distribution of this ratio in a large number of GRB afterglows of the Swift-XRT repository (Evans et al. 2009, 2010). This is presented in the next section. Along with this we also present a correlation between $L_{\text {iso }}$, the peak isotropic luminosity of the prompt emission and the afterglow X-ray luminosity $L_{X}$ at time $t=T_{\text {brk }}$, the time the X-ray afterglow resumes its more conventional decay. We finish in $\S 3$ with our conclusions and some discussion.

\section{The Prompt-to-Afterglow Flux Ratios}

Motivated by the arguments presented above, we have searched the Swift data base and compiled the ratios, $\mathcal{R}$, between the prompt and afterglow GRB fluxes. We have used fits to the average BAT light curves obtained from the Swift-XRT repository Burst Analyzer (Evans et al. 2010) extrapolated down to the $0.3-10 \mathrm{keV}$ band from fits to the XRT, as described in Racusin et al. (2009, 2011), to extract the flux at the transition between steep decline and plateau in the afterglows demonstrating that form. In figures 1 and 2 we present three specific cases of GRB transitions from the prompt to the afterglow stages with a variety of post transition behaviors. The fast decline exponents range between $\alpha \simeq-3$ and $\alpha \simeq-6$, while their transition to $\alpha \simeq-1$ happens in all cases around $T_{\text {brk }} \simeq 10^{4}$ sec. The yellow arrows show the decrease in flux between the geometric mean the highly variable prompt emission and the end of the steep decline phase.

These figures indicate that these transitions have peculiarities of their own. For example the decrease of GRB $050713 \mathrm{~A}$ is by a factor $\mathcal{R} \simeq 10^{3}$, smaller than the $m_{p} / m_{e}$ ratio; however, in the other two GRB the decrease as shown by the yellow arrows are by factors $10^{4}$ and $10^{6}$, one of them significantly larger than the $m_{p} / m_{e}$ ratio. On the other hand this last case, namely of GRB 120213A exhibits a rather peculiar two step transition

with one step decrease by a factor of $\sim 10^{4}$ followed by another one by a factor of $10^{2}$, with the horizontal lines and the yellow arrow indicating the fluxes considered by the employed algorithm. This last case indicates that, besides applying a given algorithm, one may have to scrutinize each such transition individually. 

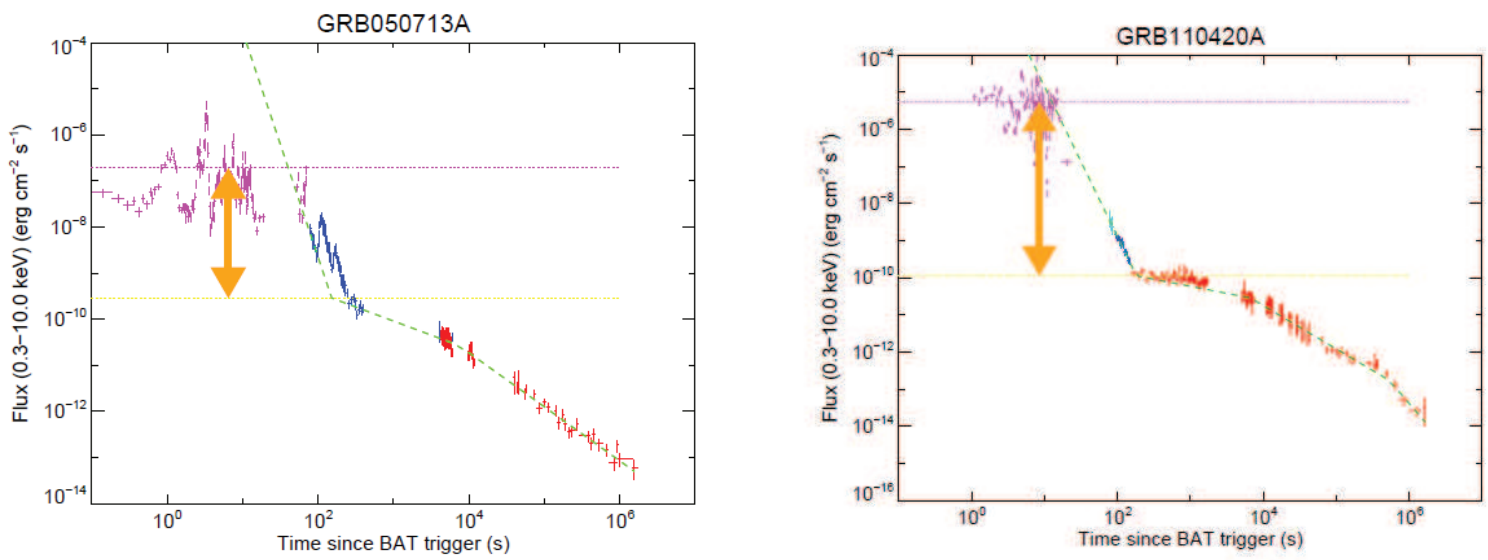

Fig. 1.- (a) The prompt to afterglow light curves of the gamma ray bursts indicated on the figure. The prompt, transition and afterglow plateau stages are apparent. The two dotted lines represent the mean prompt flux (top, purple line) and afterglow (bottom, red line) fluxes involved in computing the flux ratios of these two states.

From the point of view of the data available in search of correlations among the GRB attributes, the one proposed in SKM13 and tested herein has a clear advantage in that it involves only flux ratios rather than absolute values (whether luminosities, time lags or values of $E_{\mathrm{pk}}$ ) as is the case with many of the GRB produced correlations (e.g. the Lag-Luminosity relation, the $E_{\mathrm{pk}}-E_{\text {iso }}$, the $L_{X}-T_{\text {brk }}$. etc. correlations). As such, knowledge of the GRB redshift is not necessary, a fact that allows the compilation of a large number of bursts. The ratios of the prompt to afterglow fluxes were compiled from the Swift-XRT repository and spans the period between December 2004 and March 2014. The results of this compilation are give in figure $3 \mathrm{a}$, where we present a histogram of the prompt to afterglow flux ratios as determined by our algorithm, along with the $m_{p} / m_{e}$ ratio given by the dashed line of this figure.

The main result of our analysis is given in Fig. 3a where we present a histogram of the logarithm of the BAT-to-XRT flux ratio, $\mathcal{R}$, computed as described above along with a dashed vertical line that indicates the value of the $m_{p} / m_{e}$ ratio. The distribution exhibits a broad maximum at almost precisely the this value, indicating the presence of a characteristic ratio between the prompt and afterglow fluxes, as proposed in SKM13. The $\mathcal{R}$-distribution appears to be log-normal, though its precise shape is not easy to determine accurately. It spans 5-6 decades in $\mathcal{R}$, with a FWHM of about 2 decades and a median value of $\log \mathcal{R}$ essentially equal to that of $\log \left(m_{p} / m_{e}\right) \simeq 3.25$ and a slightly larger medium value $\left(\simeq 10^{4}\right)$ in sufficient agreement with the suggestion of Sultana, Kazanas \& Mastichiadis (2013) to merit further consideration.

As discussed above, there have been several searches for correlations between GRB attributes involving either their prompt or their afterglow emission. Amongst those of the 


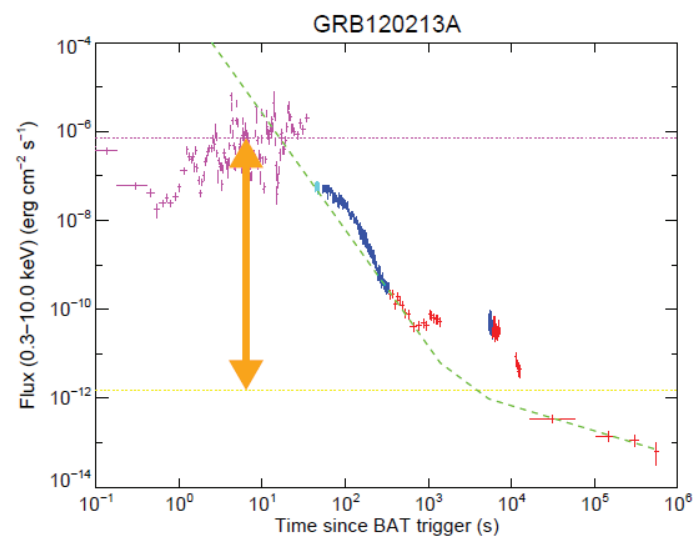

Fig. 2.- (a) The prompt to afterglow light curves of the gamma ray bursts indicated on the figure. The prompt, transition and afterglow plateau stages are apparent. The two dotted lines represent the mean prompt flux (top, purple line) and afterglow (bottom, red line) fluxes involved in computing the flux ratios of these two states.

first type we mention the Lag-Luminosity relation (Norris et al. 2000; Norris 2002; Schaefer 2007 ) and the maximum prompt emission (isotropic) luminosity $L_{\text {iso }}$ and the peak energy of the Band function $E_{\mathrm{p}}$ (Schaefer 2007; Wang, Qi \& Dai 2011). Of the correlations involving the GRB afterglow properties we mention the relation between the X-ray luminosity $L_{X}$ at the end of the plateau phase, and the rest-frame plateau-end time $T_{\text {brk }}$, beyond which the afterglow resumes the standard decline (Dainotti et al. 2008, 2010, 2013).

In Sultana, Kazanas \& Fukumura (2012) we combined these relations to show a significant correlation between the timing properties of the prompt and afterglow emissions, suggesting that these two phases are intimately connected, despite the absence of the continuity between these two phases implied by the models (Sari et al. 1998): In particular we showed that the Lag-Luminosity relation of the prompt emission extrapolates into the $L_{X}-T_{\mathrm{brk}}$ relation of their afterglow. Now, this seems to be a little strange, since the time scale associated with the Lag of the prompt emission, even though a time scale, it is of a different character than the duration of the afterglow plateau emission $T_{\text {brk }}$. With the above discussion and motivated by the histogram of Fig. 3a, we bypass the time coordinate of the relation given in Sultana. Kazanas \& Fukumura (2012) and plot in Fig. 3b the maximum prompt isotropic luminosity $L_{\text {iso }}$ vs. the X-ray luminosity of the afterglow plateau segment at the time $T_{\text {brk }}$. There appears to be a correlation between these quantities. A least squares fit gives the following relation between $L_{\text {iso }}$ and $L_{X}$

$$
\log L_{\text {iso }}=(4.04 \pm 0.10)+(1.04 \pm 0.02) \log L_{X}
$$

with correlation coefficient $\rho=0.69$. The ratio of these two quantities appears consistent with that shown in Fig. 3a. Given the difference in the choice of these samples and the slightly different properties they depict, they appear to be consistent with each other and 

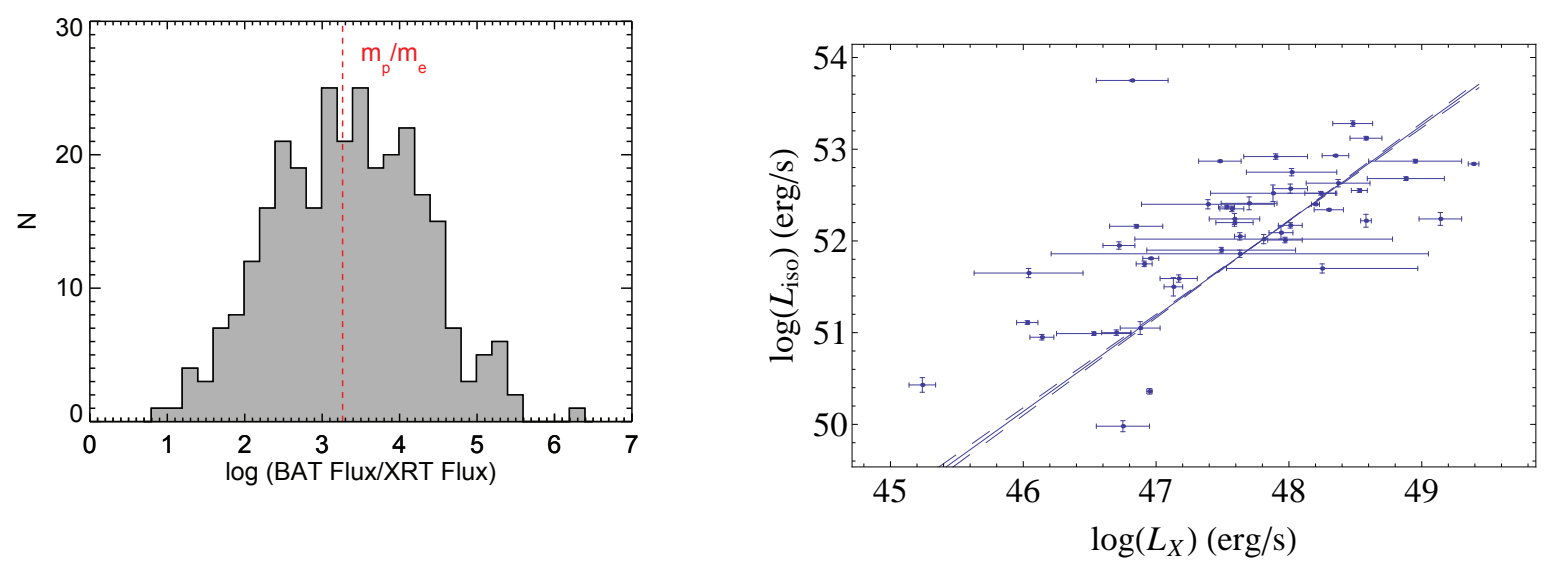

Fig. 3. - The histogram of the BAT to XRT flux ratio for a number of Swift GRB. The distribution shows clearly a preferred value for this ratio of order $\sim 10^{3}-10^{4}$. The vertical line shows also the proton to electron mass ratio $m_{p} / m_{e}$.

the general premise of the prompt to afterglow luminosity ratios.

\section{Discussion, Conclusions}

Motivated by the considerations put forward in Sultana, Kazanas \& Mastichiadis (2013) based on the tenets of the SPM model of GRB dissipation, we have compiled the flux ratios between the prompt and the afterglow plateau phases of GRB. The important point to bear in mind is that a characteristic value for the ratio $\mathcal{R}$, namely $m_{p} / m_{e}$ was given in SKM13 before the compilation of the histogram of Fig. 3a; as such, this constitutes a prediction of the model, one of the very few in the GRB field of study. A similar relation has also been found between slightly different quantities of these two GRB phases, shown in Fig. 3b, one that requires however knowledge of their redshifts.

One must note at this point that though there is a maximum in the distribution of flux ratios near the value $m_{p} / m_{e}$, the histogram and correlation of Fig. 3 have a finite width. Thus there are bursts with $\mathcal{R}$ values as large as $10^{6}$ and as low as $10^{2}$. Figure 2 shows a burst with a particularly large value of this ratio. As argued earlier, one could assign to this burst a value smaller than that given by our algorithm, given the peculiar form of its afterglow. On the other hand, if one takes into account that the apparent luminosity of a relativistically moving source can have a dependence on its Lorentz factor $\Gamma$ as strong as $\Gamma^{4}$, even a small reduction in $\Gamma$ could increase the pre-to-post prompt emission fluxes to values larger than $m_{p} / m_{e}$. Values of $\mathcal{R}<m_{p} / m_{e}$ seem to be more problematic. One possibility, 
put forward in SKM13, is that not all protons "are burnt" in prompt phase, thus reducing the flux of this stage. A related possibility is that besides the postshock Maxwellian proton distribution, of characteristic energy $\Gamma m_{p} c^{2}$, there is an additional, non-thermal, power law proton population; because this population extends to energies much higher than $\Gamma m_{p} c^{2}$, these protons continue to fulfill the pair production condition and convert their energy into pairs, as discussed in Kazanas et al. (2007), leading to a reduced value for $\mathcal{R}$. The bright bursts GRB 110731A, GRB 130427A with $\mathcal{R} \simeq 1000$ may in fact represent such cases (to keep the number of free parameters to a minimum, our earlier treatments of the SPM refrained from invoking non-thermal populations - a feature invoked at will and expediently in all GRB models; this does not mean that they are necessarily absent, however we prefer to invoke them only as a last resort). Finally, it is possible that the angle between the edge of the jet to the observer's line of sight, $\theta$, is slightly larger than $1 / \Gamma$, yielding a reduced relativistic boosting for the prompt emission. After the RBW slow-down, the smaller value of $\Gamma$ allows the observer's line of sight to "peer" directly into the relativistic outflow, thereby reducing the ratio of the pre-to-post prompt emission fluxes. Independent of the details of reason for which $\mathcal{R}<m_{p} / m_{e}$, the existence of this characteristic value in the $\mathcal{R}$-distribution, provides a new selection criterion by which we can distinguish the GRB properties (e.g. Lags, $E_{\mathrm{pk}}, L_{\text {iso }}, E_{\text {iso }}$, etc.) to get possibly novel clues into the physics of GRB emission. We hope to return to this issue in a future publication.

We conclude by pointing out that in our work, as in that of Duffell \& McFadyen (2014), the GRB afterglow plateau is associated with the dynamics of the RBW propagation rather than with the dynamics of the GRB "central engine", as is more commonly accepted; this fact should give thrust to a direction of GRB modeling orthogonal to that of heretofore. In support of the SPM is the fact that the characteristic value of the prompt to afterglow flux ratio has been predicted and the fact that this model provides a broader account of the dissipation and spectral formation in GRB. In this respect, the prompt and afterglow plateau stages are closely related, as pointed out, among others, by Sultana, Kazanas \& Mastichiadis (2013), based on the timing correlations between these two phases.

J.S. gratefully acknowledges financial support from the University of Malta during his visit at NASA-GSFC and the hospitality of the Astrophysics Science Division of GSFC. D.K. acknowledges support by Swift and Fermi GO grants. J.R. acknowledges support by the Swift and Fermi projects. 


\section{REFERENCES}

Dainotti, M. G., Cardone, V. F., \& Capozziello, S. 2008, MNRAS, 391, L79

Dainotti, M. G., Willingale, R., Capozziello, S., Cardone, V. F., \& Ostrowski, M. 2010, ApJ, 722, L215

Dainotti, M. G., Petrosian, V., Singal, J., \& Ostrowski, M. 2013, ApJ, 774, 157

Duffell, P. C. \& MacFadyen, A. I. 2014, arXiv1407.8250

Evans, P. A. et al. 2009, MNRAS, 397, 1177

Evans, P. A. et al. 2010, A\&A, 519, A102

Genet, F. and Granot, J. 2009, MNRAS, 399, 1328

Giannios, D., \& Spitkovsky, A. 2009, MNRAS, 400, 330

Gompertz, B. P., O’Brien, P. T. \& Wynn, G. A. 2014, MNRAS, 438, 240

Ioka, K., \& Nakamura, T. 2001, ApJ, 554, L163

Kazanas, D., Georganopoulos, M., \& Mastichiadis, A. 2002, ApJ, 587, L18

Kazanas, D., Mastichiadis, A., \& Georganopoulos, M. 2007, ESASP 622, 577 (also astro-ph/0612046)

Lei, H.-D., Wang, J.-Z., Lü, J. \& Zou, Y.-C. 2011, Chinese Physics Letters, 28, 129801

Mastichiadis, A., \& Kazanas, D. 2006, ApJ, 645, 416

Mastichiadis, A., \& Kazanas, D. 2008, Proc. 30th Int. Cosmic Ray Conf., eds. R. Caballero, J. C. D'Olivo, G. Medina-Tanco, L. Nellen, F. A. Sanchez, \& J. F. Valdes-Galicia, Vol. 3, 1175-1178

Mastichiadis, A., \& Kazanas, D. 2009, ApJ, 694, L54

Norris, J. P., Marani, G. F., \& Bonnell, J. T. 2000, ApJ, 534, 248

Norris, J. P. 2002, ApJ, 579, 386

Nousek, J. A. et al. 2000, ApJ, 642, 389

Petropoulou, M., Mastichiadis, A., \& Piran, T. 2011, A\&A, A76

Racusin, J. L. et al. 2009, ApJ, 698, 43

Racusin, J. L. et al. 2011, ApJ, 738, 138 
Sari, R., Piran, T., \& Narayan, R. 1998, ApJ, 497, L17

Sari, R., Piran, T., \& Halpern, J. P. 1999, ApJ, 519, L17

Schaefer, B. E. 2007, ApJ, 660, 16

Shen, R., \& Matzner C. D. 2012, ApJ, 744, 36

Sultana, J., Kazanas, D., \& Fukumura, K. 2012, ApJ, 758, 32

Sultana, J., Kazanas, D., \& Mastichiadis, A. 2013, ApJ, 779, 16 (SKM13)

Tagliaferri, G., et al. 2005, Nature, 436, 985

Wang, F.-Y., Qi, S. \& Dai, Z.-G. 2011, MNRAS, 415, 3423

Willingale, R., \& O’Brien, P. T. 2007, Phil. Tran. R. Soc. A, 365, 1179

Willingale, R., Genet, F., Granot, J. \& O’Brien, P. T., 2010, MNRAS, 403, 1296

Zhang, B. et al. 2006, ApJ, 642, 354

Zhang, B. 2007, Chin. J. As. Ap. 2007, 7, 1 